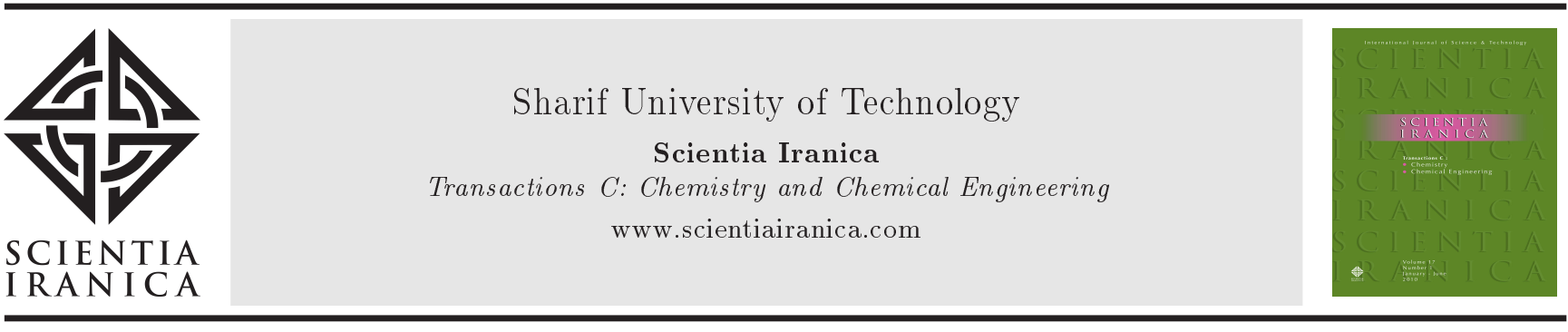

\title{
Heat transfer analysis in peristaltic slip flow with Hall and ion-slip currents
}

\author{
Q. Hussain ${ }^{a, *}$, S. Asghar ${ }^{a, b}$ and A. Alsaedi ${ }^{b}$ \\ Department of Mathematics, COMSATS Institute of Information Technology, Islamabad 44000, Pakistan. \\ Department of Mathematics, Faculty of Science, King Abdulaziz University, P.O. Box 80203, Jeddah 21589, Saudi Arabia. \\ Received 9 January 2015; received in revised form 16 January 2016; accepted 26 September 2016
}

\author{
KEYWORDS \\ Hall effect; \\ Ion-slip; \\ Slip conditions; \\ Ohmic dissipation; \\ Peristalsis; \\ Heat transfer.
}

\begin{abstract}
This work is devoted to the effects of heat transfer study on peristaltically induced flow of an electrically conducting viscous fluid in an asymmetric channel. An incompressible fluid fills the porous space in a channel. Analysis is made in the presence of Hall and ion-slip effects. The effects of velocity and thermal slips on the boundaries are considered. The problems which govern the fluid flow and heat transfer are modeled in the wave frame of reference. Exact solutions have been constructed under realistic assumptions. Important features of peristaltic motion and heat transfer are studied with the varying values of Hall parameter, ion-slip parameter, Hartman number, permeability parameter, velocity slip parameter, and thermal slip parameter. The graphical results concerning the field quantities are given in detail.

(C) 2016 Sharif University of Technology. All rights reserved.
\end{abstract}

\section{Introduction}

Peristaltic transport has gained much more attention of the investigators due to its abundant applications in physiology and industry. Such phenomenon occurs in vasomotion of small blood vessels, chyme motion in the gastrointestinal tract, movement of the ovum in the fallopian tube, swallowing of food through esophagus, urine transport from kidney to bladder through the ureter, the locomotion of some worms, venules, capillaries, etc. In addition, transportation of the sanitary fluids, finger, and roller pumps for pumping of corrosive and toxic materials and the pumping of the blood in heart-lung machines are governed by the principle of peristalsis. During the past few decades, a large number of problems related to peristalsis have been studied under different mechanical and physiological situations (see [1-6]).

The peristaltic flow of magnetohydrodynamic (MHD) fluid is significant in the study of movement

\footnotetext{
*. Corresponding author. Tel.: +92 5190642172

E-mail address: q.hussain@comsats.edu.pk (Q. Hussain)
}

of the conductive physiological fluids, analysis of blood flow through artery, blood and blood pump machines, cancer tumor treatment causing hyperthermia, bleeding reduction during surgeries, and targeted motion of drug. Moreover, it is proved that study of peristaltic flows in connection with heat transfer is also useful in many biological processes in human body, e.g. blood oxygenation, hemodialysis, metabolic process, and heat conduction in tissues. In view of the importance of heat transfer analysis in MHD flows, some authors [7-14] have focused their attention on past research.

It is found that the no-slip conditions are not suitable for thin film problems, rarefied fluid problems, polymeric fluids, and flow on multiple interfaces. The no-slip conditions for velocity and temperature are inappropriate for momentum and heat transfer in microdevices. The velocity and thermal slip conditions are adequate for the flow of liquids at the microscale level, especially when there is a lack of data on the thermal accommodation coefficient. Among the applications of microdevices, several complex microchannels arise from which microconducts of rectangular, triangular, or trapezoidal cross section are very popular 
and easier to manufacture in the microscale thermal fluid system $[15,16]$. Insignificant amount of emphasis has been devoted to the study of slip effects on peristaltically induced flows (see [17-23]).

In all the above mentioned studies, the Hall and ion-slip effects were ignored in applying the Ohm's law as they have no remarkable effect on small and moderate values of the magnetic field. However, the current trend in the application of magnetohydrodynamics is towards a strong magnetic field so that the effect of electromagnetic force is noticeable. Under these conditions, the Hall and ion-slip currents are important, and they have remarkable effects on the magnitude as well as on the direction of the current density, and consequently on the magnetic force term [24]. Existing studies on the effects of Hall and ion-slip on peristaltic flows are only by Haroun [25], Eldahab et al. [26-28], Koumy et al. [29], Uddin and Kumar [30], and Asghar et al. [31].

It seems that no attempt has been made yet for the combined effects of magnetic field, Ohmic dissipation, and Hall and ion-slip effects on the peristaltic transport of viscous fluid in a channel with velocity and thermal slip conditions. The main theme of the present investigation is to extend the analysis of Hayat et al. [31] by including the effects of Hall, ion-slip, and Ohmic dissipation on the peristaltically induced flow of electrically conducting viscous fluid through porous medium. The organization of the problem is as follows. Section 2 presents the mathematical formulation and non-dimensionalization. Section 3 refers to the mathematical results. Section 4 is prepared to analyze the effects of embedded parameters on pumping and trapping. Concluding remarks are given in Section 5 .

\section{Problem description}

We consider the two-dimensional flow in an asymmetric channel having non-conducting walls and uniform thickness $d_{1}+d_{2}$. The gap within the channel walls is assumed to be porous. Such space is filled by an incompressible and electrically conducting fluid. Two sinusoidal waves with small amplitude are traveling along the channel walls with speed $c$ and propel the fluid along the channel walls. It is important to mention that there will be no fluid motion in absence of these traveling waves. The upper and lower channel walls are subjected to thermal slip through $T_{1}$ and $T_{2}\left(T_{2}>T_{1}\right)$. A uniform magnetic field with magnetic flux density vector $\mathbf{B}=\left(0,0, B_{0}\right)$ is applied. This is assumed as the total magnetic field acting on the fluid because the induced magnetic field is neglected for the small magnetic Reynolds number. The assumption of small magnetic Reynolds number is generally true for the case of partially ionized fluids [32]. The effects of Hall and ion-slip are taken into account. In Cartesian coordinates system, the shapes of the channel walls are:

Upper wall:

$$
Y=H_{1}(X, t)=d_{1}+a_{1} \cos \left[\frac{2 \pi}{\lambda}(X-c t)\right],
$$

Lower wall:

$$
Y=H_{2}(X, t)=-d_{2}-b_{1} \cos \left[\frac{2 \pi}{\lambda}(X-c t)+\phi\right],
$$

in which $X$ and $Y$ are the axes parallel and perpendicular to the flow, $a_{1}$ and $b_{1}$ are the wave amplitude of the upper and lower walls, respectively, $\lambda$ is the wavelength, $t$ is the time, and $\phi \in[0, \pi]$ is the phase difference. It is important to note that for $\phi=0$, the channel becomes symmetric with waves out of phase; the waves are in phase for $\phi=\pi$. Furthermore, the condition $a_{1}^{2}+b_{1}^{2}+2 a_{1} b_{1} \cos \phi \leq\left(d_{1}+d_{2}\right)^{2}$ must hold so that the channel walls do not intersect with each other.

The generalized Ohm's law with Hall and ion-slip effects is:

$$
\mathbf{J}+\frac{\omega_{e} \tau_{e}}{B_{0}}(\mathbf{J} \times \mathbf{B})=\sigma(\mathbf{V} \times \mathbf{B})+\frac{\omega_{e} \tau_{e} \beta_{i}}{B_{0}^{2}}[(\mathbf{J} \times \mathbf{B}) \times \mathbf{B}],
$$

where $\mathbf{V}$ is the fluid velocity vector, $\mathbf{J}$ is the current density vector, $\omega_{e}$ is the cyclotron frequency, $\tau_{e}$ is the electron collision time, $\sigma$ is the electrical conductivity, and $\beta_{i}$ is the ion-slip parameter. Using Maxwell equations and then solving Eq. (3) for $J_{X}$ and $J_{Y}$, we obtain:

$$
\begin{aligned}
& J_{X}=\frac{\sigma B_{0}}{\left(1+\beta_{i} n^{*}\right)^{2}+n^{* 2}}\left[\left(1+\beta_{i} n^{*}\right) V+n^{*} U\right], \\
& J_{Y}=-\frac{\sigma B_{0}}{\left(1+\beta_{i} n^{*}\right)^{2}+n^{* 2}}\left[\left(1+\beta_{i} n^{*}\right) U-n^{*} V\right],
\end{aligned}
$$

where $n^{*}\left(=\omega_{e} \tau_{e}\right)$ is the Hall parameter, and $U$ and $V$ are the respective components of velocity in $X$ and $Y$-directions.

The equations of motion and heat transfer in laboratory frame $(X, Y, t)$ are:

$$
\begin{aligned}
& \frac{\partial U}{\partial X}+\frac{\partial V}{\partial Y}=0 \\
& \rho\left(\frac{\partial U}{\partial t}+U \frac{\partial U}{\partial X}+V \frac{\partial U}{\partial Y}\right)=-\frac{\partial P}{\partial X} \\
& \quad+\mu\left(\frac{\partial^{2} U}{\partial X^{2}}+\frac{\partial^{2} U}{\partial Y^{2}}\right)-\frac{\mu}{K} U+B_{0} J_{Y} \\
& \rho\left(\frac{\partial V}{\partial t}+U \frac{\partial V}{\partial X}+V \frac{\partial V}{\partial Y}\right)=-\frac{\partial P}{\partial Y} \\
& \quad+\mu\left(\frac{\partial^{2} V}{\partial X^{2}}+\frac{\partial^{2} V}{\partial Y^{2}}\right)-\frac{\mu}{K} V-B_{0} J_{X}
\end{aligned}
$$




$$
\begin{aligned}
& \rho c_{p}\left(\frac{\partial T}{\partial t}+U \frac{\partial T}{\partial X}+V \frac{\partial T}{\partial Y}\right)=\kappa\left(\frac{\partial^{2} T}{\partial X^{2}}+\frac{\partial^{2} T}{\partial Y^{2}}\right) \\
& +\Phi_{1}+\Phi_{2}+\Phi_{3}
\end{aligned}
$$

with:

$$
\begin{aligned}
& \Phi_{1}=\mu\left[2\left\{\left(\frac{\partial U}{\partial X}\right)^{2}+\left(\frac{\partial V}{\partial Y}\right)^{2}\right\}+\left(\frac{\partial U}{\partial Y}+\frac{\partial V}{\partial X}\right)^{2}\right], \\
& \Phi_{2}=\frac{\sigma B_{0}^{2}}{\left(1+\beta_{i} n^{*}\right)^{2}+n^{* 2}}\left(U^{2}+V^{2}\right), \\
& \Phi_{3}=\frac{\mu}{K}\left(U^{2}+V^{2}\right) .
\end{aligned}
$$

In Eqs. (6)-(9), $\rho$ is the fluid density, $\mu$ is the dynamic viscosity of the fluid, $K$ is the permeability of the porous medium, $P$ is the pressure, $T$ is the temperature, $\kappa$ is the thermal conductivity, $c_{p}$ is the specific heat, $\Phi_{1}$ is the viscous dissipation term, $\Phi_{2}$ is the Ohmic dissipation term, and $\Phi_{3}$ is the additional viscous dissipation term which arises due to the consolation of the porous medium.

To make the non-dimensional analysis in wave frame $(x, y)$ moving with the speed of wave $c$, we first define the components of velocity $(u, v)$ and pressure $p$ as:

$$
\begin{aligned}
& (x, y)=(X-c t, Y), \quad(u, v)=(U-c, V), \\
& p(x, y)=P(X, Y, t),
\end{aligned}
$$

and then introduce the non-dimensional quantities:

$$
\begin{array}{ll}
\hat{x}=\frac{x}{\lambda}, & \hat{y}=\frac{y}{d_{1}}, \quad \hat{u}=\frac{u}{c}, \quad \hat{v}=\frac{v}{c}, \\
\hat{t}=\frac{c t}{\lambda}, & \hat{T}=\frac{T-T_{1}}{T_{2}-T_{1}}, \quad \hat{p}=\frac{d_{1}^{2} p}{c \lambda \mu}, \\
\hat{K}=\frac{K}{d_{1}^{2}}, & \delta=\frac{d_{1}}{\lambda}, \\
\operatorname{Pr}=\frac{\mu c_{p}}{\kappa}, & \operatorname{Re}=\frac{\rho c d_{1}}{\mu}, \quad \operatorname{Er}=\frac{\sigma B_{0}^{2} d_{1}^{2}}{\mu},
\end{array}
$$

Further, the continuity Eq. (6) will be satisfied if we write the velocity components as:

$$
\hat{u}=\frac{\partial \hat{\psi}}{\partial \hat{y}}, \quad \hat{v}=-\delta \frac{\partial \hat{\psi}}{\partial \hat{x}},
$$

where $\hat{\psi}=\hat{\psi}(\hat{x}, \hat{y})$ is the stream function.

Invoking Eqs. (10)-(12) into Eqs. (7)-(9) and adopting the long wavelength procedure, one obtains:

$$
0=\frac{\partial^{4} \psi}{\partial y^{4}}-\left(\frac{M^{2}\left(1+\beta_{i} n^{*}\right)}{\left(1+\beta_{i} n^{*}\right)^{2}+n^{* 2}}+\frac{1}{K}\right) \frac{\partial^{2} \psi}{\partial y^{2}},
$$

$$
\begin{aligned}
0= & -\frac{d p}{d x}+\frac{\partial^{3} \psi}{\partial y^{3}} \\
& -\left(\frac{M^{2}\left(1+\beta_{i} n^{*}\right)}{\left(1+\beta_{i} n^{*}\right)^{2}+n^{* 2}}+\frac{1}{K}\right)\left(\frac{\partial \psi}{\partial y}+1\right), \\
0= & \frac{1}{\operatorname{Pr}} \frac{\partial^{2} T}{\partial y^{2}} \\
& +\operatorname{Ec}\left\{\left(\frac{M^{2}}{\left(1+\beta_{i} n^{*}\right)^{2}+n^{* 2}}+\frac{1}{K}\right)\left(\frac{\partial \psi}{\partial y}+1\right)^{2}\right. \\
& \left.+\left(\frac{\partial^{2} \psi}{\partial y^{2}}\right)^{2}\right\},
\end{aligned}
$$

and $\partial p / \partial y=0$ which indicates that $p \neq p(y)$. In Eqs. (13)-(15), we denote Hartman number by $M$, permeability parameter by $K$, Prandtl number by Pr, and Eckert number by Ec. In writing above equations, the hats have been suppressed.

The relevant boundary conditions in wave frame are:

$$
\begin{aligned}
& \psi=\frac{F}{2}, \quad \frac{\partial \psi}{\partial y}+\beta_{v} \frac{\partial^{2} \psi}{\partial y^{2}}=-1, \quad T+\beta_{t} \frac{\partial T}{\partial y}=0 \\
& \text { at } y=h_{1}(x), \\
& \psi=-\frac{F}{2}, \quad \frac{\partial \psi}{\partial y}-\beta_{v} \frac{\partial^{2} \psi}{\partial y^{2}}=-1, \quad T-\beta_{t} \frac{\partial T}{\partial y}=1, \\
& \text { at } y=h_{2}(x),
\end{aligned}
$$

in which $\beta_{v}$ and $\beta_{t}$ are the dimensionless slip parameters corresponding to slip conditions for velocity and temperature. These are defined by:

$$
\beta_{v}=\xi / d_{1}, \quad \beta_{t}=\eta / d_{1},
$$

where $\xi$ and $\eta$ are the dimensional velocity and thermal slip parameters with dimension of length.

Now, the peristaltic walls $h_{1}(x)$ and $h_{2}(x)$ in dimensionless form takes the form:

$$
\begin{aligned}
& h_{1}(x)=1+a \cos [2 \pi x], \\
& h_{2}(x)=-d-b \cos [2 \pi x+\phi],
\end{aligned}
$$

where amplitude ratios $a\left(=a_{1} / d_{1}\right)$ and $b\left(=b_{1} / d_{1}\right)$, channel width ratio $d\left(=d_{2} / d_{1}\right)$, and $\phi$ satisfy the inequality condition $a^{2}+b^{2}+2 a b \cos \phi \leq(1+d)^{2}$.

The dimensionless time-mean flow rate $F$ in wave frame is defined by:

$$
F=\int_{h_{2}(x)}^{h_{1}(x)} \frac{\partial \psi}{\partial y} d y
$$

which can be further related to the time-mean flow rate 
$Q$ in the laboratory frame by:

$$
Q=F+1+d \text {. }
$$

The pressure rise per wavelength and frictional forces at $y=h_{1}(x)$ and $y=h_{2}(x)$, respectively, are defined by:

$$
\begin{aligned}
& \Delta P_{\lambda}=\int_{0}^{1}\left(\frac{d p}{d x}\right) d x, \\
& F_{\lambda}^{(1)}=\int_{0}^{1}-h_{1}^{2}\left(\frac{d p}{d x}\right) d x, \\
& F_{\lambda}^{(2)}=\int_{0}^{1}-h_{2}^{2}\left(\frac{d p}{d x}\right) d x .
\end{aligned}
$$

The heat transfer coefficient at $y=h_{1}(x)$ is given by:

$$
Z=\frac{\partial h_{1}}{\partial x} \frac{\partial T}{\partial y} \text {. }
$$

\section{Mathematical results}

Solutions of Eqs. (13)-(15), satisfying the conditions (16) and (17), may be expressed by the equations shown in Box I. as follows:

\section{Graphical results and discussion}

In this section, we study the important features of the fluid motion and heat transfer with respect to the embedded parameters such as Hall parameter, $n^{*}$; ion-slip parameter, $\beta_{i}$; Hartman number, $\mathrm{M}$; permeability parameter, $K$; velocity slip parameter; $\beta_{v}$; thermal slip parameter, $\beta_{t}$; and Brinkman number, Br. For this purpose, we have plotted pressure rise per wavelength, $\Delta P_{\lambda}$, in Figure 1, frictional forces,
$F_{\lambda}^{(s)}(s=1,2)$, in Figure 2, axial pressure gradient, $d p / d x$, in Figure 3, velocity field, $u$, in Figure 4, temperature field, $T$, in Figure 5 , and streamlines in Figures 6-8. Table 1 is prepared to see the variation in heat transfer coefficient, $Z$. It is important to mention that due to the complexity of the integrals in Eqs. (21) and (22), numerical integration is performed by the built-in routine NIntegrate in MATHEMATICA 8.

We have calculated pressure rise per wavelength depending $\Delta P_{\lambda}$ on mean flow rate $Q(=F+1+d)$ for various values of $n^{*}, \beta_{i}, \mathrm{M}, K$ and $\beta_{v}$. The results are shown in Figure 1 . We observed from Figure 1(a) and (b) that an increase in each of $n^{*}$ and $\beta_{i}$ decreases the pumping rate in retrograde pumping region $\left(\Delta P_{\lambda}>0, Q<0\right)$, while there is an increase in the pumping rate in co-pumping region $\left(\Delta P_{\lambda}<0, Q>0\right)$. In peristaltic pumping region $\left(\Delta P_{\lambda}>0, Q>0\right)$, the pumping rate decreases with the increase of $n^{*}$ and $\beta_{i}$ up to certain value of the flow rate which is approximately equal to 0.13 . For this value of the flow rate, the pumping lines intersect with each other and when $Q>0.13$, the pumping rate starts increasing with the increase of $n^{*}$ and $\beta_{i}$. Furthermore, we have also calculated the maximum pressure $P_{0}$ required to produce zero flow rate, and free pumping flux, $Q_{0}$, corresponds to $\Delta P_{\lambda}=0$. Numerical values of $P_{0}$ and $Q_{0}$ are displayed along with the graphs. It is noted that the value of the maximum pressure, $P_{0}$, against which peristalsis works as a pump is greater in the absence of Hall and ionslip currents $\left(n^{*}=0, \beta_{i}=0\right)$; it is lower when Hall and ion-slip currents are present. The free pumping flux $Q_{0}$ increases with an increase in the values of

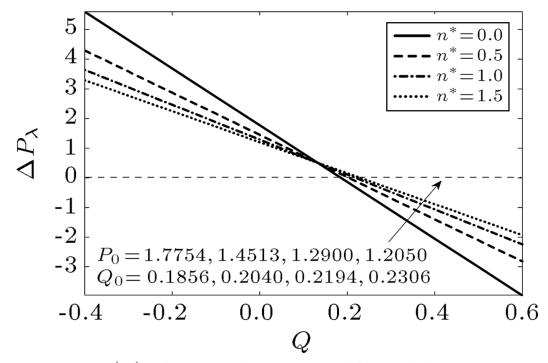

(a) $\beta_{i}=1, \beta_{v}=0.1, K=1, M=3$

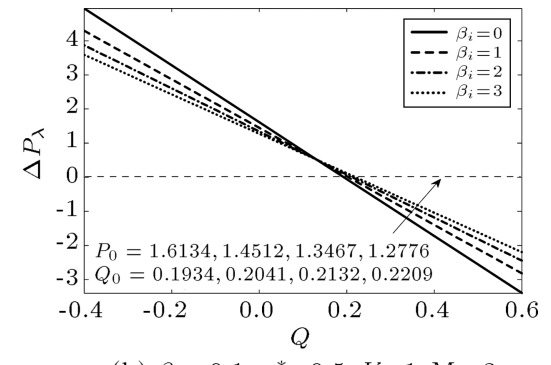

(b) $\beta_{v}=0.1, n^{*}=0.5, K=1, \mathrm{M}=3$

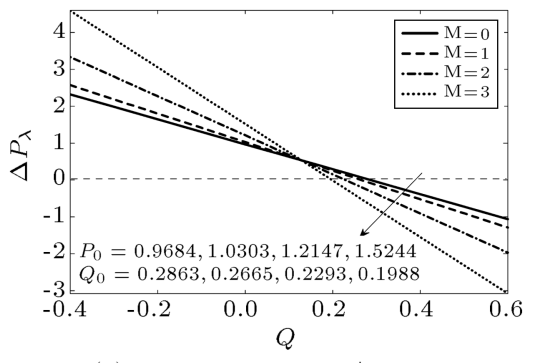

(c) $\beta_{i}=0.5, \beta_{v}=0.1, n^{*}=0.5, K=1$

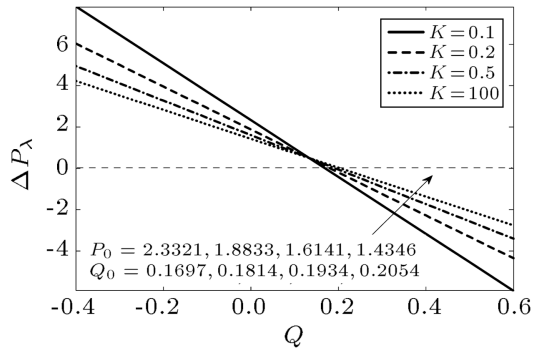

(d) $\beta_{i}=0.5, \beta_{v}=0.1, n^{*}=0.5, \mathrm{M}=3$

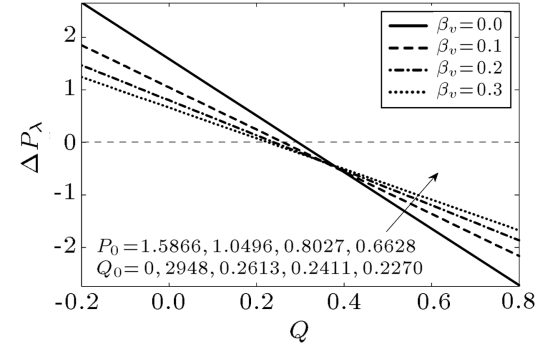

(e) $\beta_{i}=1, n^{*}=0.1, K=1, \mathrm{M}=1$

Figure 1. Plots of pressure rise per wavelength for $a=0.2, b=0.5, d=0.7$, and $\phi=\pi / 4$. 


$$
\begin{aligned}
& \psi=K_{1}+K_{2} y+K_{3} \cosh [\Omega y]+K_{4} \sinh [\Omega y] \\
& \frac{d p}{d x}=-\frac{\Omega^{3}\left(h_{1}-h_{2}+F\right)\left(\cosh \left[\frac{\Omega\left(h_{1}-h_{2}\right)}{2}\right]+\Omega \beta_{v} \sinh \left[\frac{\Omega\left(h_{1}-h_{2}\right)}{2}\right]\right)}{\Omega\left(h_{1}-h_{2}\right) \cosh \left[\frac{\Omega\left(h_{1}-h_{2}\right)}{2}\right]-\left(2-\Omega^{2} \beta_{v}\left(h_{1}-h_{2}\right)\right) \sinh \left[\frac{\Omega\left(h_{1}-h_{2}\right)}{2}\right]} \\
& T=A_{5}+A_{6} y+\operatorname{Br} g(y)
\end{aligned}
$$

with:

$$
\begin{aligned}
& \Omega=\sqrt{\frac{M^{2}\left(1+\beta_{i} n^{*}\right)}{\left(1+\beta_{i} n^{*}\right)^{2}+n^{* 2}}+\frac{1}{K}}, \quad \Lambda=\sqrt{\frac{M^{2}}{\left(1+\beta_{i} n^{*}\right)^{2}+n^{* 2}}+\frac{1}{K}}, \\
& g(y)=-\frac{1}{4}\left(2 \Lambda^{2}\left(1+K_{2}\right)^{2}+\Omega^{2}\left(\Omega^{2}-\Lambda^{2}\right)\left(K_{3}^{2}-K_{4}^{2}\right)\right) y^{2}-\frac{1}{8} K_{4}\left(\Lambda^{2} K_{4} \cosh [2 \Omega y]+2\left(\Omega^{2}+\Lambda^{2}\right) K_{3} \sinh [2 \Omega y]\right) \\
& -2 \frac{\Lambda^{2}}{\Omega}\left(1+K_{2}\right)\left(K_{4} \cosh [\Omega y]+K_{3} \sinh [\Omega y]\right)-\frac{1}{8}\left(\Omega^{2} K_{4}^{2}+\left(\Omega^{2}+\Lambda^{2}\right) K_{3}^{2}\right) \cosh [2 \Omega y] \\
& K_{1}=-\frac{\left(h_{1}+h_{2}\right)\left(F \Omega \cosh \left[\frac{\left(h_{1}-h_{2}\right) M}{2}\right]+\left(2+F \Omega^{2} \beta_{v}\right) \sinh \left[\frac{\left(h_{1}-h_{2}\right) \Omega}{2}\right]\right)}{2\left(\left(h_{1}-h_{2}\right) \Omega \cosh \left[\frac{\left(h_{1}-h_{2}\right) \Omega}{2}\right]-\left(2-\left(h_{1}-h_{2}\right) \Omega^{2} \beta_{v}\right) \sinh \left[\frac{\left(h_{1}-h_{2}\right) \Omega}{2}\right]\right)}, \\
& K_{2}=\frac{F \Omega \cosh \left[\frac{\left(h_{1}-h_{2}\right) \Omega}{2}\right]+\left(2+F \Omega^{2} \beta_{v}\right) \sinh \left[\frac{\left(h_{1}-h_{2}\right) \Omega}{2}\right]}{\left(h_{1}-h_{2}\right) \Omega \cosh \left[\frac{\left(h_{1}-h_{2}\right) \Omega}{2}\right]-\left(2-\left(h_{1}-h_{2}\right) \Omega^{2} \beta_{v}\right) \sinh \left[\frac{\left(h_{1}-h_{2}\right) \Omega}{2}\right]}, \\
& K_{3}=\frac{\left(h_{1}-h_{2}+F\right) \sinh \left[\frac{\left(h_{1}+h_{2}\right) \Omega}{2}\right]}{\left(h_{1}-h_{2}\right) \Omega \cosh \left[\frac{\left(h_{1}-h_{2}\right) \Omega}{2}\right]-\left(2-\left(h_{1}-h_{2}\right) \Omega^{2} \beta_{v}\right) \sinh \left[\frac{\left(h_{1}-h_{2}\right) \Omega}{2}\right]} \text {, } \\
& K_{4}=-\frac{\left(h_{1}-h_{2}+F\right) \cosh \left[\frac{\left(h_{1}+h_{2}\right) \Omega}{2}\right]}{\left(h_{1}-h_{2}\right) \Omega \cosh \left[\frac{\left(h_{1}-h_{2}\right) \Omega}{2}\right]-\left(2-\left(h_{1}-h_{2}\right) \Omega^{2} \beta_{v}\right) \sinh \left[\frac{\left(h_{1}-h_{2}\right) \Omega}{2}\right]}, \\
& K_{5}=\frac{\operatorname{Br}\left(h_{2}-\beta_{t}\right)\left(g\left[h_{1}\right]+\beta_{t} \frac{\partial g}{\partial y}\left[h_{1}\right]\right)+\left(h_{1}+\beta_{t}\right)\left(1-\operatorname{Br}\left(g\left[h_{2}\right]-\beta_{t} \frac{\partial g}{\partial y}\left[h_{2}\right]\right)\right)}{h_{1}-h_{2}+2 \beta_{t}} \\
& K_{6}=-\frac{1+\operatorname{Br}\left(g\left[h_{1}\right]-g\left[h_{2}\right]+\beta_{t}\left(\frac{\partial g}{\partial y}\left[h_{1}\right]+\frac{\partial g}{\partial y}\left[h_{2}\right]\right)\right)}{h_{1}-h_{2}+2 \beta_{t}}
\end{aligned}
$$

in which $\operatorname{Br}(=\operatorname{PrEc})$ represents the Brinkman number.

\section{Box I}

Hall and ion-slip parameters. Figure 1(c) depicts that the effects of $\mathrm{M}$ on $\Delta P_{\lambda}$ are inversely proportional to the effects of $n^{*}$ and $\beta_{i}$, whereas the effects of $K$ on $\Delta P_{\lambda}$ are directly proportional to the effects of $n^{*}$ and $\beta_{i}$ as illustrated in Figure $1(\mathrm{~d})$. However, the value of mean flow rate $Q$ against which pumping lines intersect with each other remains the same. Figure 1(e) explains the influence of velocity slip parameter $\beta_{v}$ on $\Delta P_{\lambda}$. It is clear that an increase in $\beta_{v}$ results in a decrease in pumping rate for $Q<0.37$, whilst it increases with increasing $\beta_{v}$ for $Q>0.37$. Further, the maximum pressure $P_{0}$ and free pumping flux $Q_{0}$ decrease when $\beta_{v}$ increases. Here, we can conclude that peristaltic pumping region $\left(\Delta P_{\lambda}>0, Q>0\right)$ becomes narrow when velocity slip at the boundary walls is larger. 


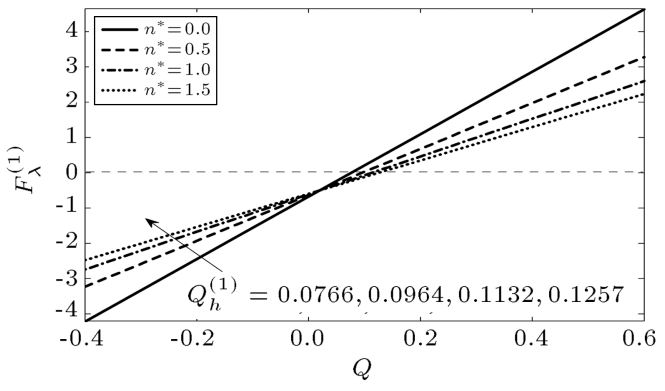

(a) $\beta_{i}=1, \beta_{v}=0.1, K=1, \mathrm{M}=3$

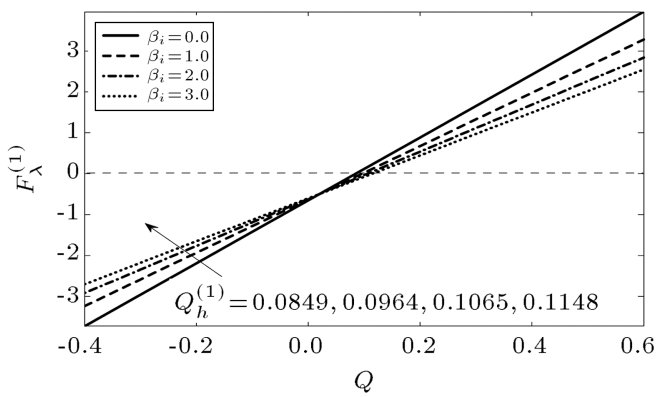

(c) $\beta_{v}=0.1, n^{*}=0.5, K=1, \mathrm{M}=3$

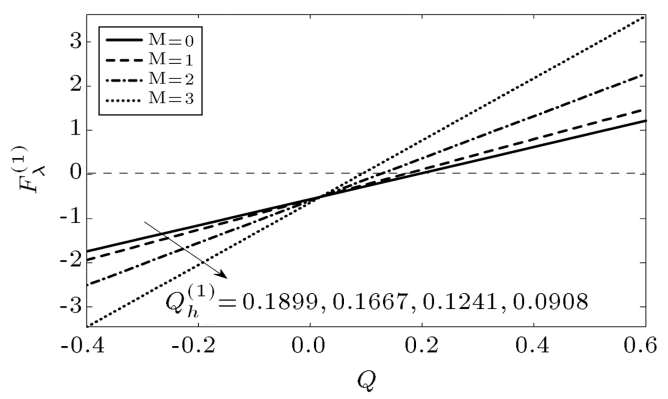

(e) $\beta_{i}=0.5, \beta_{v}=0.1, n^{*}=0.5, K=1$

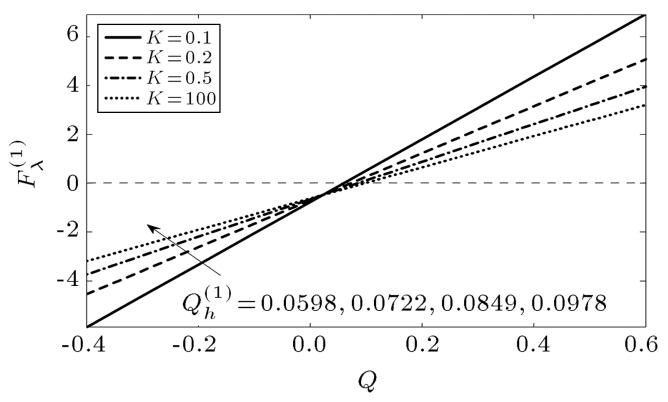

(g) $\beta_{i}=0.5, \beta_{v}=0.1, n^{*}=0.5, \mathrm{M}=3$

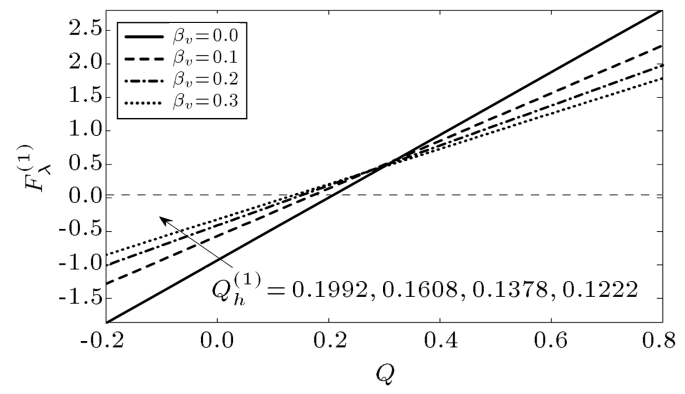

(i) $\beta_{i}=1, n^{*}=0.1, K=1, \mathrm{M}=1$

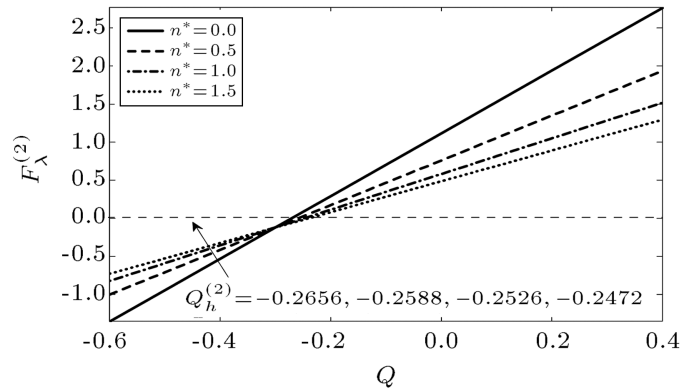

(b) $\beta_{i}=1, \beta_{v}=0.1, K=1, \mathrm{M}=3$

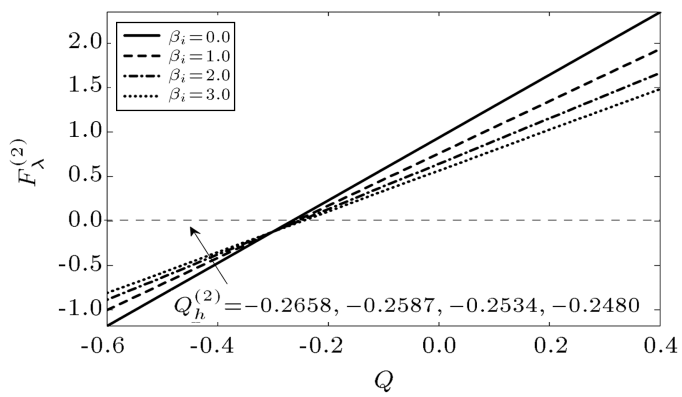

(d) $\beta_{v}=0.1, n^{*}=0.5, K=1, \mathrm{M}=3$

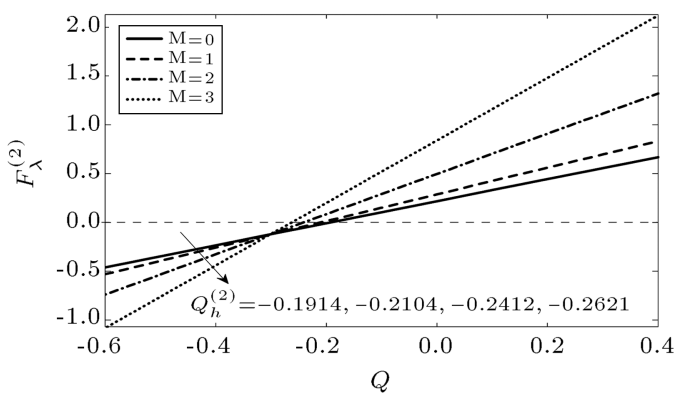

(f) $\beta_{i}=0.5, \beta_{v}=0.1, n^{*}=0.5, K=1$

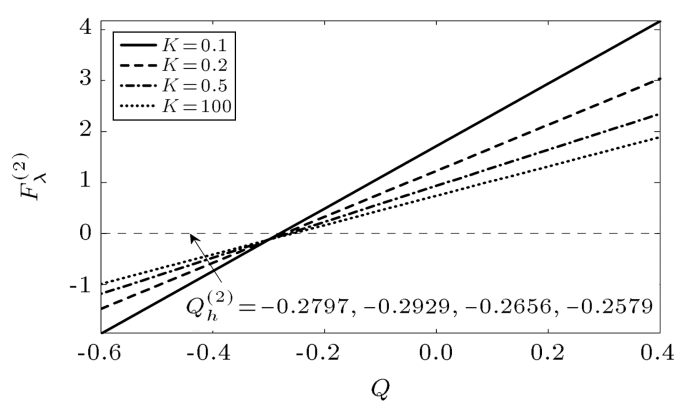

(h) $\beta_{i}=0.5, \beta_{v}=0.1, n^{*}=0.5, \mathrm{M}=3$

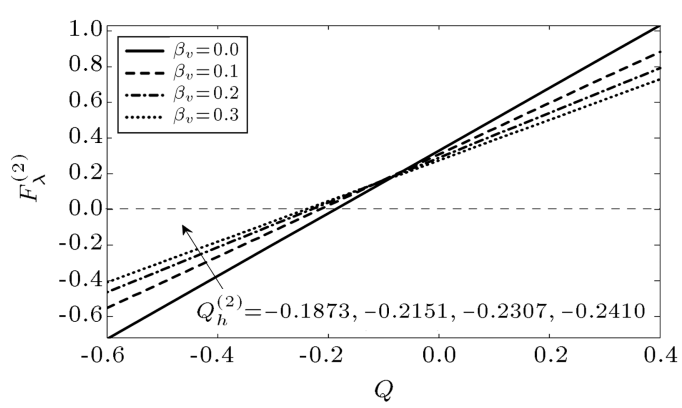

(j) $\beta_{i}=1, n^{*}=0.1, K=1, \mathrm{M}=1$

Figure 2. Plots of frictional forces at $y=h_{1}(x)$ for $a=0.2, b=0.5, d=0.7$, and $\phi=\pi / 4$. 


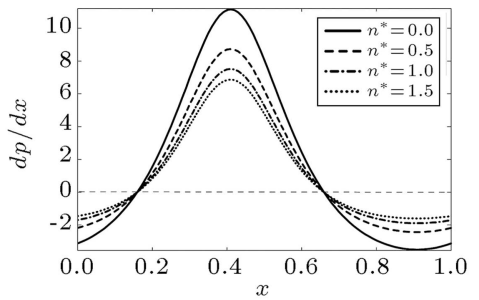

(a) $\beta_{i}=1, \beta_{v}=0.1, K=1, \mathrm{M}=3$

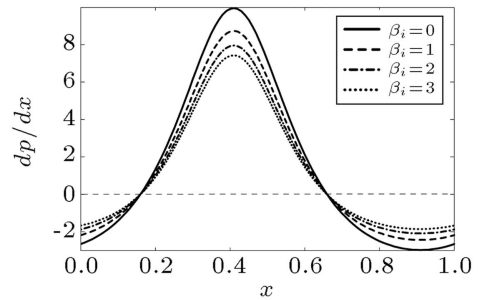

(b) $\beta_{v}=0.1, n^{*}=0.5, K=1, \mathrm{M}=3$

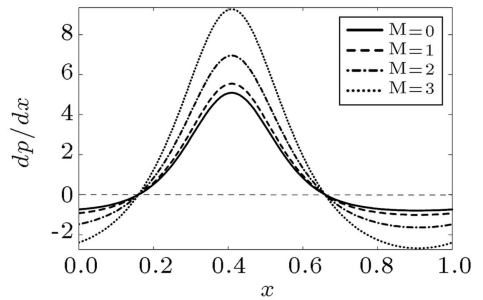

(c) $\beta_{i}=0.5, \beta_{v}=0.1, n^{*}=0.5, K=1$

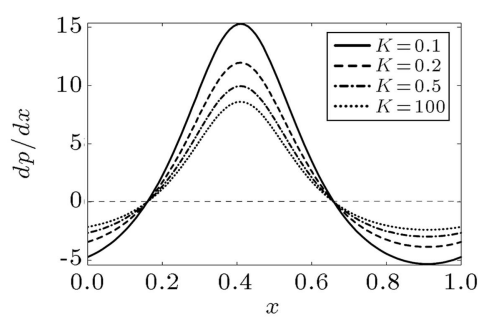

(d) $\beta_{i}=0.5, \beta_{v}=0.1, n^{*}=0.5, \mathrm{M}=3$

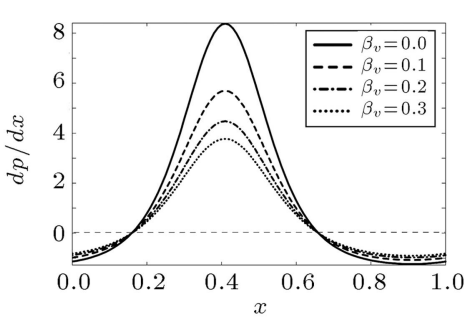

(e) $\beta_{i}=1, n^{*}=0.1, K=1, \mathrm{M}=1$

Figure 3. Plots of axial pressure gradient for $a=0.2, b=0.5, d=0.7, \phi=\pi / 4$, and $Q=0$.

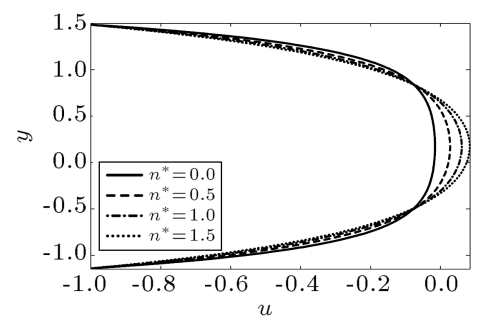

(a) $\beta_{i}=1, \beta_{v}=0, K=1, M=4$

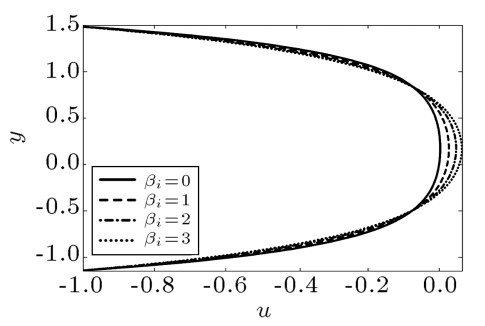

(b) $\beta_{v}=0, n^{*}=0.5, K=1, \mathrm{M}=4$

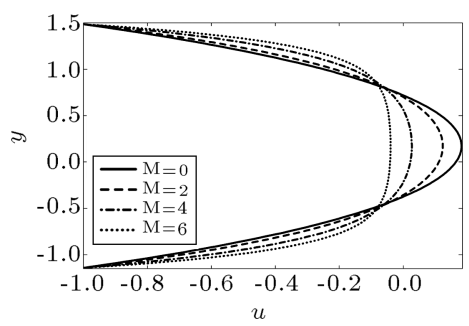

(c) $\beta_{i}=1, \beta_{v}=0, n^{*}=0.5, K=1$

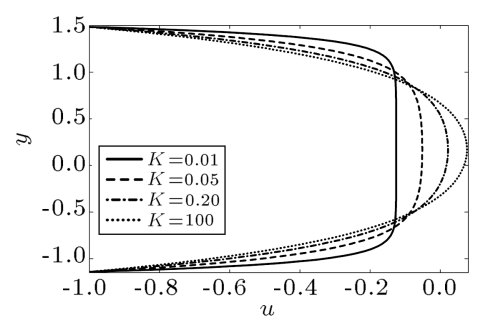

(d) $\beta_{i}=0.5, \beta_{v}=0, n^{*}=0.5, \mathrm{M}=3$

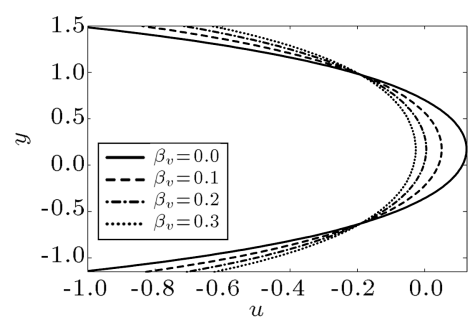

(e) $\beta_{i}=1, n^{*}=0.5, K=1, \mathrm{M}=2$

Figure 4. Plots of velocity profile for $a=0.6, b=0.9, d=1, \phi=\pi / 4, x=0.1$, and $Q=1.5$.

The behavior of frictional forces $F_{\lambda}^{(1)}$ at $y=h_{1}(x)$ and $F_{\lambda}^{(2)}$ at $y=h_{2}(x)$ against time mean flow rate $Q$ for different values of $n^{*}, \beta_{i}, \mathrm{M}, K$, and $\beta_{v}$ are illustrated in Figure 2. Here, we observed that the behavior of frictional forces $F_{\lambda}^{(s)}(s=1,2)$ is quite opposite to that of the observed for the case of $\Delta P_{\lambda}$ (comparison of Figure 1 and Figure 2). Furthermore, there also exists a critical value $Q_{h}^{(s)}$ corresponding to $F_{\lambda}^{(s)}=0$. Below this critical value, the friction force $F_{\lambda}^{(s)}(s=1,2)$ creates resistance to the flow and above which it supports the fluid to drive. The critical values $Q_{h}^{(s)}(s=1,2)$ are shown with the graphs. We noted that the critical value $Q_{h}^{(1)}$ is positive and increases when one of $n^{*}, \beta_{i}$, and $K$ is increased, whereas it decreases with an increase in each of $\mathrm{M}$ and $\beta_{v}$. Similar behavior has been seen for the case of $Q_{h}^{(2)}$. However, this critical value corresponds to negative flow rate.

To see the variation in axial pressure gradient $d p / d x$ for one cycle of wave $x \in[0,1]$, we have sketched Figure 3 for different values of the parameters $n^{*}, \beta_{i}$, $\mathrm{M}, K$, and $\beta_{v}$. Figure 3 exhibits that the pressure gradient has maximum value at the narrowest position of the channel, i.e. $x=0.41$, which means that a much larger amount of pressure gradient is needed to pass the fluid through this part of the channel. Furthermore, the pressure gradient is negative when $x \in[0,0.16] \cup[0.66,1]$. In this region, $d p / d x$, known as favorable pressure gradient, assists the fluid flow. On the other hand, when $x \in[0.16,0.66]$, the pressure gradient $d p / d x$ becomes positive and resists the fluid flow. This is known as adverse pressure gradient. 


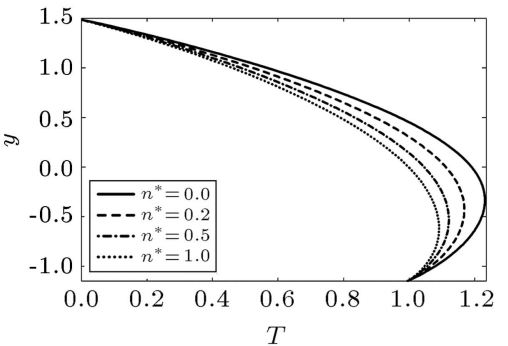

(a) $\beta_{i}=1, \beta_{v}=0, \beta_{t}=0, \mathrm{Br}=0.5, K=1, \mathrm{M}=1$

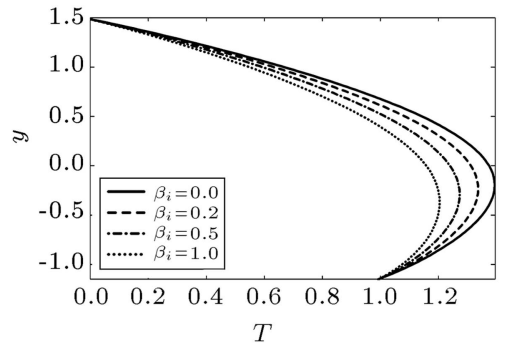

(b) $\beta_{v}=0, \beta_{t}=0, n^{*}=0.5, \mathrm{Br}=0.5, K=1, \mathrm{M}=1.5$

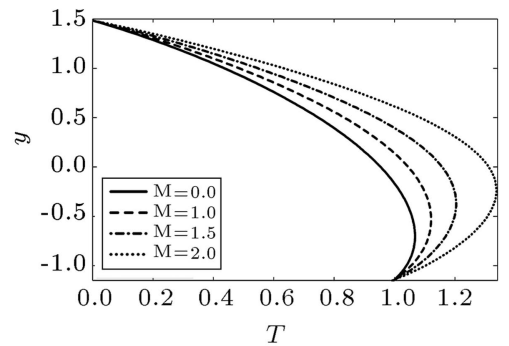

(c) $\beta_{i}=1, \beta_{v}=0, \beta_{t}=0, n^{*}=0.5, \mathrm{Br}=0.5, K=1$

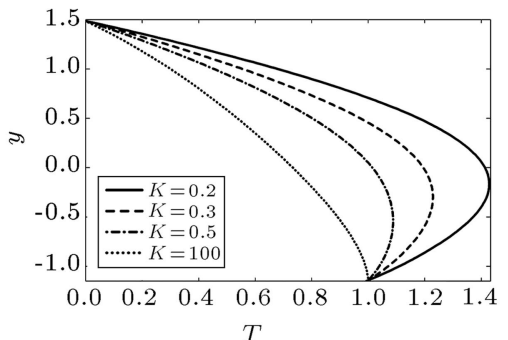

(d) $\beta_{i}=0.5, \beta_{v}=0, \beta_{t}=0, n^{*}=0.5, \mathrm{Br}=0.3, \mathrm{M}=1$

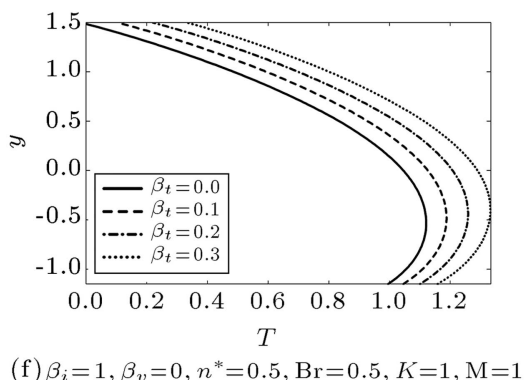

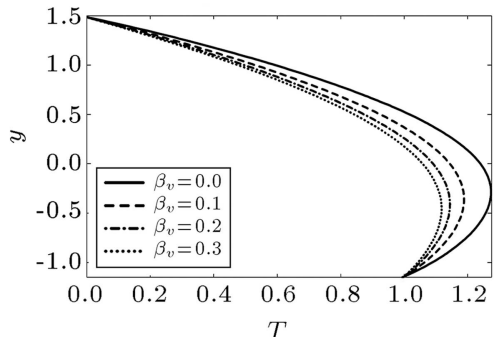

(e) $\beta_{i}=0.5, \beta_{t}=0, n^{*}=0.5, \mathrm{Br}=0.5, K=1, \mathrm{M}=1.5$

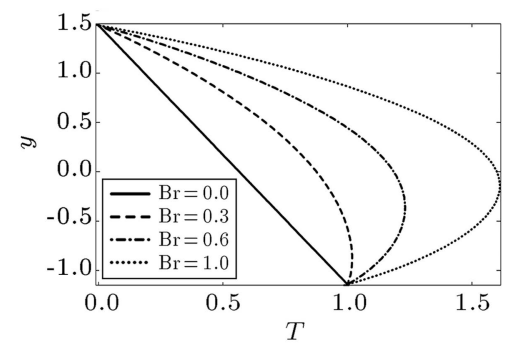

(g) $\beta_{i}=0.5, \beta_{v}=0, \beta_{t}=0, n^{*}=0.5, \mathrm{Br}=0.5, K=1, M=1$

Figure 5. Plots of temperature profile for $a=0.6, b=0.9, d=1.0, \phi=\pi / 4, x=0.1$, and $Q=1$.

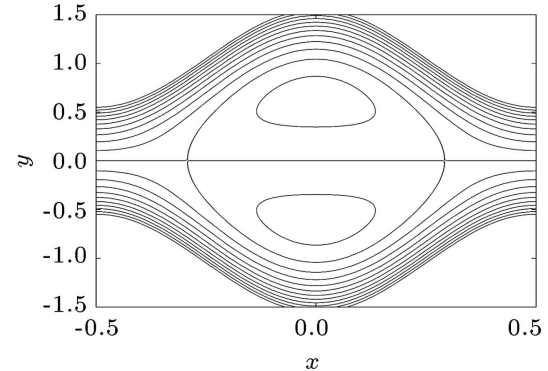

(a) $n^{*}=0, \phi=0$

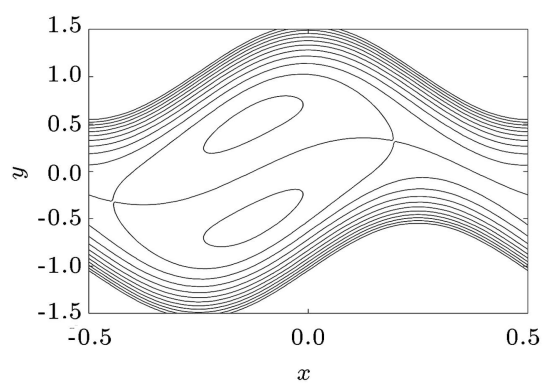

(d) $n^{*}=0.3, \phi=\pi / 2$

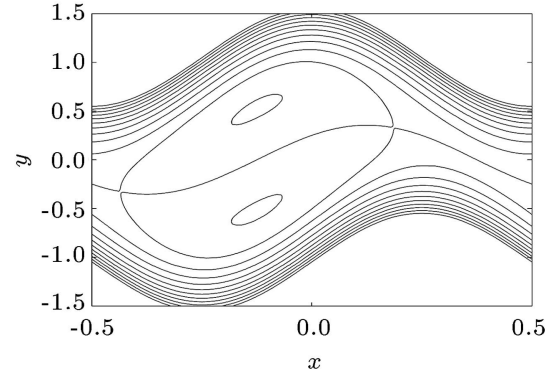

(b) $n^{*}=0, \phi=\pi / 2$

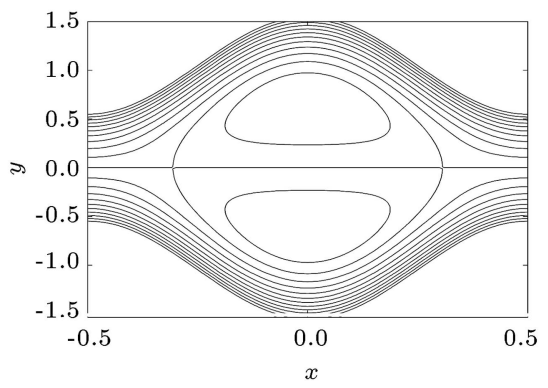

(e) $n^{*}=0.8, \phi=0$

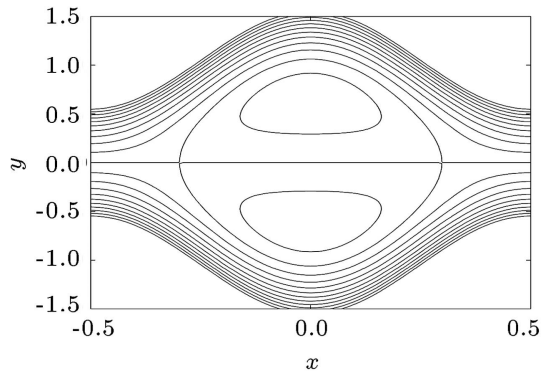

(c) $n^{*}=0.3, \phi=0$

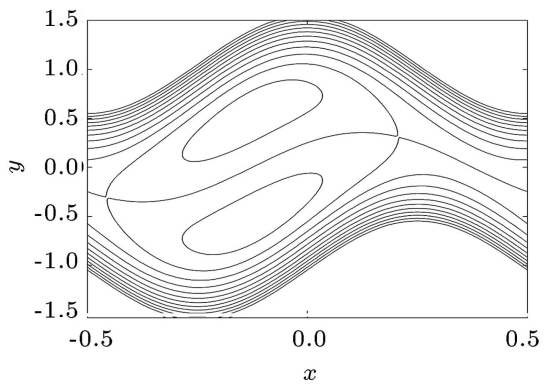

(f) $n^{*}=0.8, \phi=\pi / 2$

Figure 6. Streamlines for various values of Hall parameter $n^{*}$ and phase difference $\phi$ with $a=0.5, b=0.5, d=1$, $Q=1.5, M=2, K=1, \beta_{i}=0.5$, and $\beta_{v}=0.01$. 


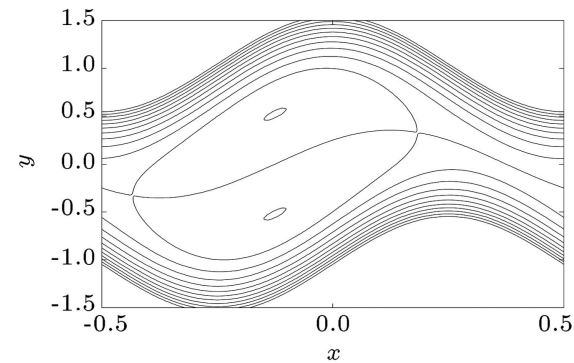

(a) $K=0.5, \beta_{i}=0$

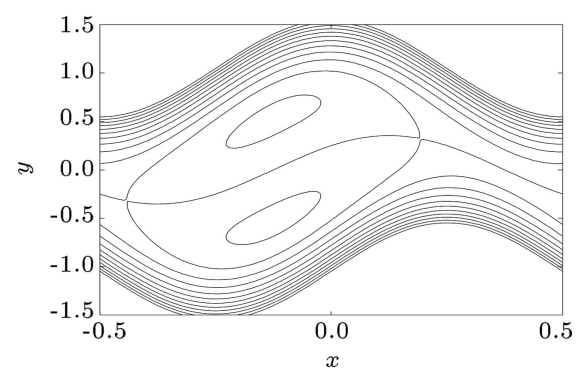

(c) $K=0.8, \beta_{i}=0$

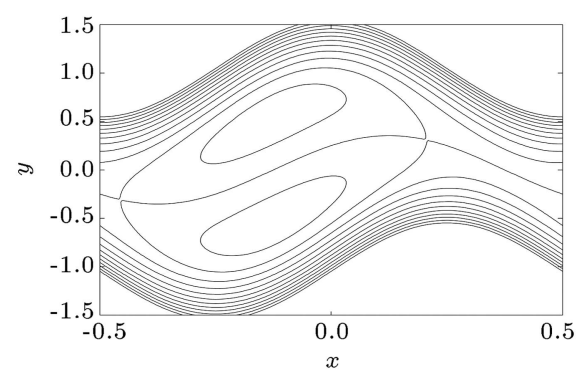

(e) $K=100, \beta_{i}=0$

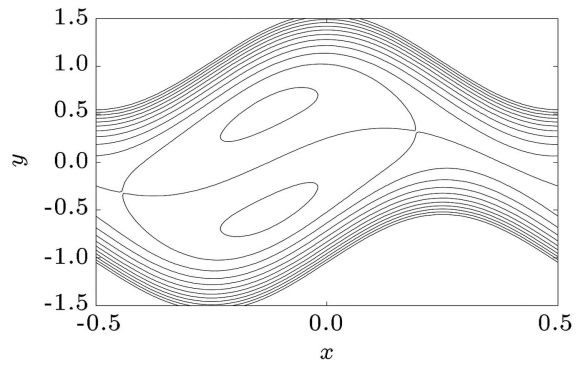

(b) $K=0.5, \beta_{i}=1$

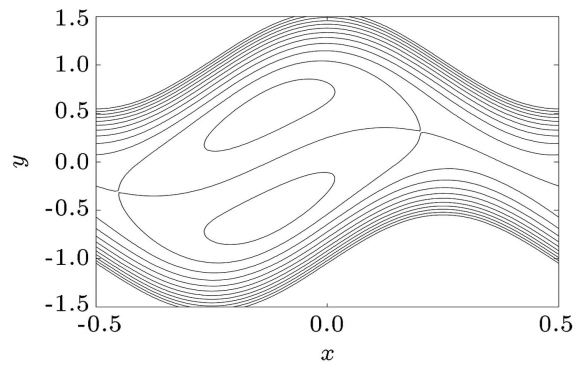

(d) $K=0.8, \beta_{i}=1$

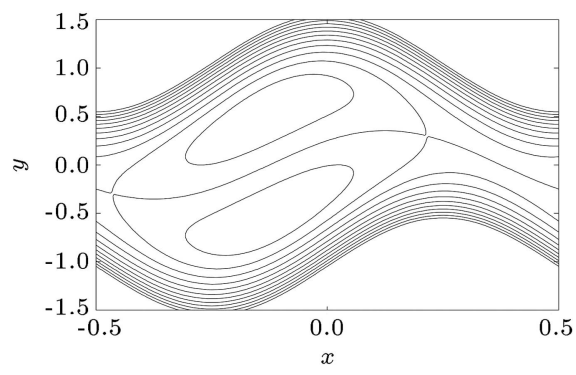

(f) $K=100, \beta_{i}=1$

Figure 7. Streamlines for various values of porous medium parameter $K$ and ion-slip parameter $\beta_{i}$ with $a=0.5, b=0.5$, $d=1, \phi=\pi / 2, Q=1.5, M=2, \beta_{v}=0.01$, and $n^{*}=0.5$.

The Hall parameter $n^{*}$ and ion-slip parameter $\beta_{i}$ have similar impact on $d p / d x$ which can be seen from the Figures 3(a) and 3(b), respectively. We observed that adverse pressure gradient decreases by increasing $n^{*}$ and $\beta_{i}$, while favorable pressure gradient increases with an increase in $n^{*}$ and $\beta_{i}$. The influence of $\mathrm{M}$ on pressure gradient $d p / d x$ is illustrated in Figure 3(c). It is clear from the figure that an increase in Hartman number, $\mathrm{M}$, corresponds to an increase in adverse pressure gradient, whereas there is a decrease in the favorable pressure gradient. It can be seen in Figure 3(d) that effects of $K$ on $d p / d x$ are reverse when compared with the effects of $\mathrm{M}$. The variation in $d p / d x$ for different values of velocity slip parameter, $\beta_{v}$, are given in Figure 3(e). It is noticed that adverse pressure gradient decreases when $\beta_{v}$ is increased. However, the favorable pressure gradient increases. Furthermore, the effect of $\beta_{v}$ on adverse pressure gradient is more pronounced than that of the favorable pressure gradient.

The velocity field $u$ against $y$ for different values of $n^{*}, \beta_{i}, \mathrm{M}, K$, and $\beta_{v}$ is shown in Figure 4. The observations regarding the effects of Hall parameter $n^{*}$, ion-slip parameter $\beta_{i}$, and Hartman number $\mathrm{M}$ (given in Figure 4(a)-(c)) are already established in [31] and need not to be repeated. The results presented in Figure 4(d) predict the disturbance in the velocity field $u$ with $K$. It is clear that the velocity $u$ is less parabolic for smaller values of the permeability parameter $K$. This is the case when a thin boundary layer is formed in the surrounding of the channel walls, and flow away from the walls is plug-like. However, the velocity $u$ becomes parabolic when $K$ is increased and sharing extends from the channel walls to the whole flow domain. An increase in the velocity slip parameter, $\beta_{v}$, results in an increase in velocity $u$ at the channel walls, whereas it decreases at the center part of the channel with an increase in $\beta_{v}$ (see Figure $4(\mathrm{e})$ ).

Our next consideration will be the temperature field, $T$, that is plotted against $y$ for different values of $n^{*}, \beta_{i}, \mathrm{M}, K, \beta_{v}, \beta_{t}$, and $\operatorname{Br}$ (see Figure 5). Out of these parameters, the effects of $n^{*}, \beta_{i}$, and $M$ (Figure 5(a)-(c)) are qualitatively the same as given in [31]. The subsequent discussion is made for other parameters. The temperature field $T$ with an increase in $K$ is shown in Figure $5(\mathrm{~d})$. It reveals that the effect of $K$ is to increase the fluid temperature $T$ in the 


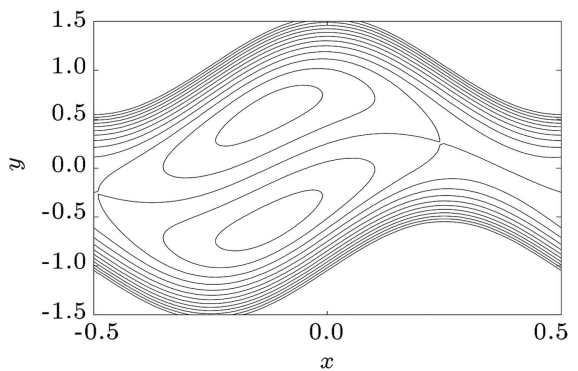

(a) $\mathrm{M}=0, \beta_{v}=0.00$

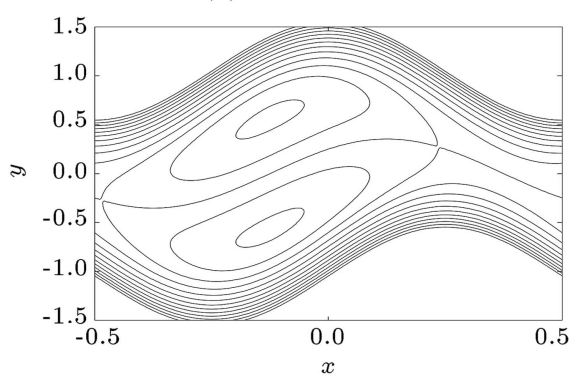

(c) $\mathrm{M}=1, \beta_{v}=0.00$

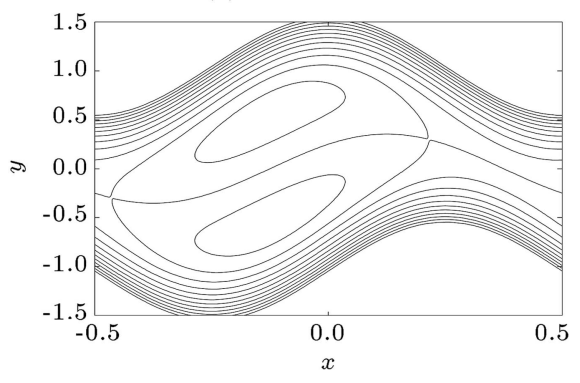

(e) $\mathrm{M}=2, \beta_{v}=0.00$

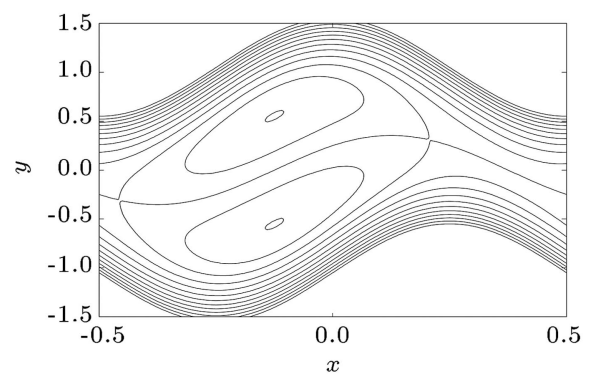

(b) $\mathrm{M}=0, \beta_{v}=0.03$

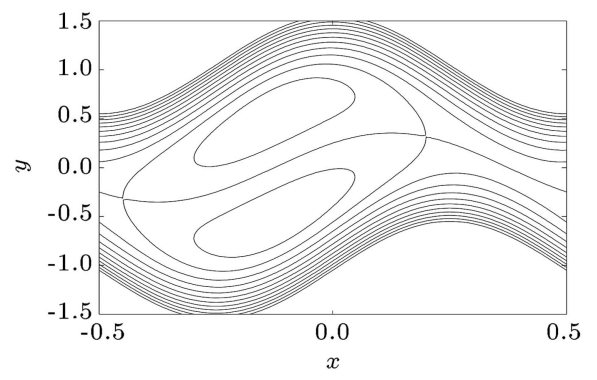

(d) $\mathrm{M}=1, \beta_{v}=0.03$

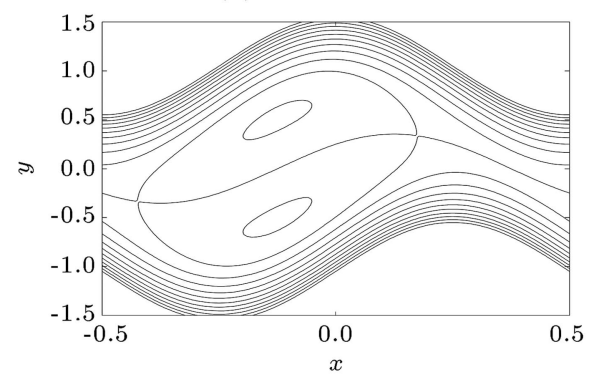

(f) $\mathrm{M}=2, \beta_{v}=0.03$

Figure 8. Streamlines for various values of Hartman number $M$ and velocity-slip parameter $\beta_{v}$ with $a=0.5, b=0.5$, $d=1, \phi=\pi / 2, Q=1.5, K=1, \beta_{i}=0.5$, and $n^{*}=0.5$.

whole flow field. Influence of velocity slip parameter $\beta_{v}$ on temperature field, $T$, is illustrated in Figure $5(\mathrm{e})$. Here, one can see that the temperature $T$ decreases when the slip at the boundary is increased. Moreover, the variations in temperature $T$ are larger for smaller values of $\beta_{v}$. On the other hand, Figure $5(\mathrm{f})$ shows that an increase in the thermal slip parameter, $\beta_{t}$, increases $T$ at the channel walls and at the center of the channel. The effect of $\mathrm{Br}$ is to increase the temperature $T$ as illustrated in Figure 5(g).

Table 1 is prepared to analyze the variation in heat transfer coefficient $Z$ at fixed cross section $x=0.1$ for different values of $n^{*}, \beta_{i}, \mathrm{M}, K, \beta_{v}, \beta_{t}$, and $\mathrm{Br}$. It is observed that $Z$ is maximum in absence of Hall current, and it decreases with an increase in Hall parameter $n^{*}$. Similar observation can be made regarding the effects of ion-slip parameter $\beta_{i}$ on $Z$. An increase in $M$ results in an increase in the value of heat transfer coefficient $Z$. The permeability parameter $K$ has an opposite effect on $Z$ when compared with the previous case of $M$. The heat transfer coefficient $Z$ decreases both for increasing velocity and thermal slip parameters. However, the rate at which $Z$ decreases is better for the case of velocity slip when compared with the thermal slip. An increase in $Z$ is observed with an increase in Brinkman number Br.

We have sketched Figures 6-8 to discuss the influence of $n^{*}, \beta_{i}, K, \mathrm{M}$, and $\beta_{v}$ on the trapped bolus. Figure 6 shows that trapped bolus is symmetric in the symmetric channel. As $\phi$ increases, the channel becomes asymmetric and the symmetry of the bolus is destroyed. Moreover, the volume of the trapped bolus is small for the case of asymmetric channel when compared to the case of symmetric channel. An increment in $n^{*}$ yields an increase in the volume of the trapped bolus. Figure 7 illustrates that the volume of the trapped bolus increases with an increase in each of $\beta_{i}$ and $K$. However, Figure 8 depicts the opposite effects on the trapped bolus with an increase of $\mathrm{M}$ and $\beta_{v}$.

\section{Closing remarks}

We have presented the heat transfer analysis in peristaltically induced flow of magnetohydrodynamic fluid in the presence of Hall and ion-slip currents. The 
Table 1. Variation of heat transfer coefficient $Z$ for $a=0.5, b=0.6, d=1, \phi=\pi / 4, x=0.1$, and $Q=1$.

\begin{tabular}{|c|c|c|c|}
\hline $\begin{array}{c}\text { Parameters } \\
\text { (fixed values) }\end{array}$ & $\begin{array}{r}\mathrm{F} \\
\text { (va }\end{array}$ & $\begin{array}{l}\text { neters } \\
\text { values) }\end{array}$ & $Z$ \\
\hline \multirow{4}{*}{$\beta_{i}=1, \beta_{v}=0, \beta_{t}=0.1, K=1, \mathrm{M}=3, \mathrm{Br}=0.5$} & \multirow{4}{*}{$n^{*}$} & 0 & 6.2376 \\
\hline & & 0.3 & 4.4032 \\
\hline & & 0.6 & 3.4710 \\
\hline & & 1 & 2.8574 \\
\hline \multirow{4}{*}{$\beta_{v}=0, \beta_{t}=0.1, n^{*}=1, K=1, \mathrm{M}=3, \mathrm{Br}=0.5$} & \multirow{4}{*}{$\beta_{i}$} & 0 & 4.1383 \\
\hline & & 0.3 & 3.5929 \\
\hline & & 0.6 & 3.2050 \\
\hline & & 1 & 2.8574 \\
\hline \multirow{4}{*}{$\beta_{i}=0.5, \beta_{v}=0.1, \beta_{t}=0.1, n^{*}=0.5, K=1, \mathrm{Br}=0.5$} & \multirow{4}{*}{ M } & 0 & 1.6481 \\
\hline & & 1 & 1.8890 \\
\hline & & 2 & 2.6062 \\
\hline & & 3 & 3.7884 \\
\hline \multirow{4}{*}{$\beta_{i}=0.5, \beta_{v}=0.1, \beta_{t}=0, n^{*}=0.5, \mathrm{M}=1, \mathrm{Br}=0.5$} & \multirow{4}{*}{$K$} & 0.1 & 5.8134 \\
\hline & & 0.2 & 3.6812 \\
\hline & & 0.5 & 2.3823 \\
\hline & & 100 & 1.5056 \\
\hline \multirow{4}{*}{$\beta_{i}=0.5, \beta_{t}=0, n^{*}=0.5, K=1, \mathrm{M}=1, \mathrm{Br}=0.5$} & \multirow{4}{*}{$\beta_{v}$} & 0 & 2.3011 \\
\hline & & 0.1 & 1.9438 \\
\hline & & 0.2 & 1.7607 \\
\hline & & 0.3 & 1.6545 \\
\hline \multirow{4}{*}{$\beta_{i}=0.5, \beta_{v}=0, n^{*}=0.5, K=1, \mathrm{M}=1, \mathrm{Br}=0.5$} & \multirow{4}{*}{$\beta_{t}$} & 0 & 2.3011 \\
\hline & & 0.1 & 2.2464 \\
\hline & & 0.2 & 2.1991 \\
\hline & & 0.3 & 2.1580 \\
\hline \multirow{4}{*}{$\beta_{i}=0.5, \beta_{v}=0, \beta_{t}=0, n^{*}=0.5, K=1, \mathrm{M}=1$} & \multirow{4}{*}{$\mathrm{Br}$} & 0 & 0.7391 \\
\hline & & 0.3 & 1.6763 \\
\hline & & 0.6 & 2.6136 \\
\hline & & 1 & 3.8632 \\
\hline
\end{tabular}

problems associated with the fluid flow and heat transfer are modeled in wave frame of reference. Then, exact solutions have been constructed. The results are displayed through the graphs in order to interpret the influence of various interesting parameters. The main findings of this article are listed as follows:

- The maximum pressure, $P_{0}$, against which peristalsis works as pump decreases with an increase in each of Hall parameter $n^{*}$, ion-slip parameter $\beta_{i}$, permeability parameter $K$, and velocity slip parameter $\beta_{v}$. However, it decreases when Hartman number $\mathrm{M}$ is increased;

- The critical values, $Q_{0}, Q_{h}^{(1)}$, and $Q_{h}^{(2)}$, increase when there is an increase in $n^{*}, \beta_{i}$, and $K$. However, the opposite effects are seen for the cases of $\mathrm{M}$ and $\beta_{v}$

- The effects of $n^{*}, \beta_{i}, K$, and $\beta_{v}$ are to decrease the adverse pressure gradient, while the effect of $\mathrm{M}$ is to increase it. Furthermore, the favorable pressure gradient shows the opposite behavior when compared with the adverse pressure gradient;

- An increase in $K$ results in an increase at the center of the channel and a decrease near the channel walls;

- The velocity $u$ decreases around the centerline of the channel when $\beta_{v}$ is increased, whilst it increases along the channel walls;

- The transfer of heat is reduced by an increase in $K$ 
and $\beta_{v}$. However, it increases due to an increase in both $\beta_{t}$ and $\mathrm{Br}$;

- An increase in $n^{*}, \beta_{i}, K, \beta_{v}$, and $\beta_{t}$ leads to a reduction in the value of the heat transfer coefficient $Z$. On the other hand, $Z$ increases for increasing $M$ and $\mathrm{Br}$;

- An increase in $n^{*}, \beta_{i}$, and $K$ results in an increase in the volume of the trapped bolus, while the effects of $M$ and $\beta_{v}$ on trapped bolus are the opposite.

\section{References}

1. Zendehbudi, G.R. and Moayeri, M.S. "Analysis of pulsatile flowthrough stenotic arteries", Sci. Iran., 6, pp. 131-142 (1999).

2. Afifi, N.A.S. and Gad, N.S. "Interaction of peristaltic flow with pulsatile magnto-fluid through a porous medium", Acta Mech., 149, pp. 229-237 (2001).

3. Elshehawey, E.F. and Husseny, S.Z.A. "Peristaltic transport of a magneto-fluid with porous boundaries", Appl. Math. Comput., 129, pp. 421-440 (2002).

4. Meheimer, Kh.S. "Peristaltic flow of blood under the effect of magnetic field in a non-uniform channel", Appl. Math. Comput., 153, pp. 763-777 (2004).

5. Elshahed, M. and Haroun, M.H. "Peristaltic transport of Johson-Segalman fluid under effect of a magnetic field", Math. Probl. Eng., 6, pp. 663-667 (2005).

6. Vajravelu, K., Sreenadh, S. and Babu, V.R. "Peristaltic pumping of a Herschel-Bulkley fluid in a channel", Appl. Math. Comput., 169, pp. 726-735 (2005).

7. Vajravelu, K., Radhakrishnamacharya, G. and Radhakrishnamurty, V. "Peristaltic flow and heat transfer in a vertical porous annulus with long wave approximation", Int. J. Nonlin. Mech., 42, pp. 754-759 (2007).

8. Radhakrishnamacharya, G. and Srinivasulu, Ch. "Influence of wall properties on peristaltic transport with heat transfer", C.R. Mecanique., 335, pp. 369-373 (2007).

9. Mekheimer, Kh.S. and Abd elmaboud, Y. "The influence of heat transfer and magnetic field on peristaltic transport of a Newtonian fluid in a vertical annulus: Application of an endoscope", Phys. Lett. A., 372, pp. 1657-1665 (2008).

10. Srinivas, S. and Kothandapani, M. "Peristaltic transport in an asymmetric channel with heat transfer, A note", Int. Commun. Heat Mass Trans., 35, pp. 514$522(2008)$

11. Ali, N., Sajid, M., Javed, T. and Abbas, Z. "Heat transfer analysis of peristaltic flow in a curved channel", Int. J. Heat Mass Trans., 53, pp. 3319-3325 (2010).

12. Mozayyeni, H.R. and Rahimi, A.B. "Mixed convection in cylindrical annulus with rotating outer cylinder and constant magnetic field with an effect in the radial direction", Sci. Iran. B, 19, pp. 91-105 (2012).
13. Tripathi, D. "Study of transient peristaltic heat flow through a finite porous channel", Math. Comput. Model., 57, pp. 1270-1283 (2013).

14. Hussain, Q., Hayat, T., Asghar, S. and Alsulami, H.H. "Mixed convective peristaltic transport in a vertical channel with Robin's condition", J. Braz. Soc. Mech. Sci. Eng., 36, pp. 681-695 (2014).

15. Martin, M.J. and Boyd, I.D. "Momentum and heat transfer in laminar boundary layer with slip flow", $J$. Thermophy. Heat Trans., 20, pp. 710-719 (2006).

16. Zhu, X. and Liao, Q. "Heat transfer for laminar slip flow in a microchannel of arbitrary cross section with complex", Appl. Therm. Eng., 26, pp. 1246-1256 (2006).

17. Ali, N., Hussain, Q., Hayat, T. and Asghar, S. "Slip effects on the peristaltic transport of MHD fluid with variable viscosity", Phys. Lett. A, 372, pp. 1477-1489 (2008).

18. Sobh, A.M. "Interaction of couple stresses and slip flow on peristaltic transport in uniform and nonuniform channels", Turk. J. Eng. Environ. Sci., 32, pp. 117123 (2008).

19. Ebaid, A. "Effects of magnetic field and wall slip conditions on the peristaltic transport of a Newtonian fluid in an asymmetric channel", Phys. Lett. A, 372, pp. 4493-4499 (2008).

20. Srinivas, S., Gayathri, R. and Kothandapani, M. "The influence of slip conditions, wall properties and heat transfer on MHD peristaltic transport", Comput. Phys. Commun., 180, pp. 2115-2122 (2009).

21. Yıldırım, A. and Sezer, S.A. "Effects of partial slip on the peristaltic flow of a MHD Newtonian fluid in an asymmetric channel", Math. Comput. Model., 52, pp. 618-625 (2010).

22. Hayat, T., Hussain, Q., Qureshi, M.U., Ali, N. and Hendi, A.A. "Influence of slip condition on the peristaltic transport with heat transfer: an exact solution", Int. J. Numer. Meth. Fluids, 67, pp. 19441959 (2011).

23. Eldesoky, M.I. "Influence of slip condition on peristaltic transport of a compressible maxwell fluid through porous medium in a tube", Int. J. Appl. Math. Mech., 8, pp. 99-117 (2012).

24. Cramer, K. and Pai, S.I., Magnetofluid Dynamics for Engineers and Applied Physicists, MCGraw-Hill Book Co. (1973).

25. Haroun, M.H. "A mathematical study of hall effects on peristaltic transport of hydromagnetic flow through a porous medium", J. Porous Media, 10, pp. 687-700 (2007).

26. Abo-Eldahab, E.M., Barakat, E.I. and Nowar, K. "Effects of Hall and ion-slip currents on peristaltic transport of a couple stress fluid", Int. J. Appl. Math. Phys., 2, pp. 145-157 (2010).

27. Abo-Eldahab, E.M., Barakat, E.I. and Nowar, K.I. "Hall currents and ion-slip effects on the MHD 
peristaltic transport", Int. J. Appl. Math. Phys., 2, pp. 113-123 (2010).

28. Abo-Eldahab, E., Barakat, E. and Nowar, K. "Hall currents and heat transfer effects on peristaltic transport in a vertical asymmetric channel through a porous medium", Math. Probl. Eng., 2012, Article ID. 840203 (2012).

29. El-Koumy, S.R., Barakat, E.I. and Abdelsalam, S.I. "Hall and porous boundaries effects on peristaltic transport through porous medium of a Maxwell model", Trans. Porous Media, 94, pp. 643-658 (2012).

30. Uddin, Z. and Kumar, M. "Hall and ion-slip effect on MHD boundary layer flow of a micro polar fluid past a wedge", Sci. Iran. B, 20, pp. 467-476 (2013).

31. Asghar, S., Hussain, Q., Hayat, T. and Alsaadi, F. "Hall and ion-slip effects on peristaltic flow and heat transfer analysis with Ohmic heating", Appl. Math. Mech.-Eng. Ed., 35, pp. 1509-1524 (2014).

32. Cowling, T.G., Magnetohydrodynamics, Interscience Publisher, Inc, New York (1957).

\section{Biographies}

Qumar Hussain is an Assistant Professor in the Department of Mathematics at COMSATS Institute of Information Technology (CIIT), Islamabad, Pakistan. He received his PhD degree from CIIT in 2015. His area of research is theoretical study of peristaltic motion of Newtonian and non-Newtonian fluids. He has 8 years of teaching and research experience. He is the author of several research articles published in ISI recognized journals.
Saleem Asghar is working as Eminent Professor at COMSATS Institute of Information Technology Islamabad. His current research interests are in Theoretical Fluid Dynamics. He has teaching and research experience spanning more than four decades. He has published significantly in International journals. He has supervised more than twenty PhD scholars. His services have been recognized by the government of Pakistan in terms of civil awards. He has been Fulbright fellow (USA), DAAD fellow (Germany), and visiting mathematician (ICTP, Italy).

Ahmed Alsaedi obtained his $\mathrm{PhD}$ degree from Swansea University (UK) in 2002. He has a broad experience of research in applied mathematics. His fields of interest include dynamical systems, nonlinear analysis involving ordinary differential equations, fractional differential equations, boundary value problems, mathematical modeling, biomathematics, and Newtonian and Non-Newtonian fluid mechanics. He has published several articles in peer-reviewed journals. He has supervised several MS students and executed many research projects successfully. He is a reviewer of several international journals. He served as the Chairman of the Mathematics Department at KAU. Presently, he is serving as a director of the research program at KAU. Under his great leadership, this program is running quite successfully, and it has attracted a large number of highly rated researchers and distinguished professors from all over the world. He is also the head of NAAM international research group at KAU. 